\title{
Novel balance rehabilitation and training apparatus to improve functional balance
}

\author{
David M. Koceja, PhD; ${ }^{*}$ Jeffrey S. Greiwe, $\mathbf{P h D}^{\mathbf{2}}$ \\ ${ }^{1}$ Department of Kinesiology, School of Public Health, Indiana University, Bloomington, IN; ${ }^{2}$ Research and Develop- \\ ment, Balancemetrics, Fort Thomas, $K Y$
}

\begin{abstract}
A new balance rehabilitation and training apparatus has been developed to allow a balance-impaired person to cope with his or her fear of falling while safely and independently performing exercises necessary to improve functional balance. The apparatus consists of a stable platform where the user stands and a vertical structure that supports free-floating handles that the user holds with both hands while performing various exercises. The purpose of study 1 was to determine whether this new apparatus significantly alters the biological postural control system, and the purpose of study 2 was to document the benefits of balance training using the apparatus. Study 1 was a randomized repeated-measures design with six healthy adult subjects (mean age $=35.5$ yr), and study 2 was a 4 wk intervention case study with a generally healthy 63-yr-old individual. The results suggest that postural sway characteristics and the cortical and proprioceptive feedback were not limited when using the apparatus. We also observed improvements in balance control and postural stability with 4 wk of training with the apparatus. These results support that the apparatus could be an effective tool to help individuals safely and independently perform balance exercises while potentially preventing falls and minimizing fear of falling.
\end{abstract}

Key words: aging, balance, balance impaired, cortical, fall prevention, fall risk, freesway, geriatric, postural sway, proprioceptive.

\section{INTRODUCTION}

It is no secret that America's population is aging, and individuals $65 \mathrm{yr}$ and older are the fastest growing age group in our nation. In 2010, approximately 40 million
Americans were $65 \mathrm{yr}$ and older, and this age group is projected to increase to approximately 72 million by 2030 [1]. As a result of unprecedented success in health maintenance, these individuals are expected to live longer than ever. The average American female can expect to live to approximately $80.4 \mathrm{yr}$ and the average American male to approximately 75.2 yr [2]. With our aging population and increased life expectancy comes one very practical concern: quality of life. A critical component to quality of life is the independence granted by mobility. Hence, a primary focus of research in neuromuscular rehabilitation should be directed toward attenuating the age-related declines in motor function.

It is a well-known fact that the quality of motor performance deteriorates with age. Age-related motor deficits are associated with deleterious changes in peripheral as well as in central nervous system parameters. The gradual decline in motor performance with age leads to one of the greatest threats to the quality of life of older adults, which is the risk of falling. When the elderly fall, major fractures, permanent disability, and even death can result. Despite continued efforts of National Institutes of Health-funded research to reduce the occurrence of falls

Abbreviations: ANOVA $=$ analysis of variance, $\mathrm{COP}=$ center of pressure, DFA = detrended fluctuation analysis.

*Address all correspondence to David M. Koceja, PhD; Department of Kinesiology, School of Public Health, Indiana University, Bloomington, IN 47405; 812-855-7302. Email: koceja@indiana.edu

http://dx.doi.org/10.1682/JRRD.2014.03.0082 
in the elderly, the rate of falls is projected to continue to increase [3]. The cost of fall-related injuries in those aged over 65 yr was a substantial \$19 billion in 2000 [4], and it is projected to cost $\$ 54.9$ billion by the year 2020 [3].

Research suggests that falls are not a normal part of aging, and most falls can be prevented with implementation of effective strategies [5]. The current challenge is to develop a safe means to effectively implement these strategies in order to help the aging population. One strategy that has been identified includes regular exercise to improve balance, strength, and flexibility [5]. Unfortunately, the very fear of falling itself often prevents implementation of these proven programs for a large percentage of the population [6]. A patient who is fearful of falling holds onto stable objects as a crutch to overcome his or her fear. Convention teaches and supports the idea that holding onto a stable object is a good thing, and physical therapy routines often incorporate some form of stable support. However, fixed support has been demonstrated to limit postural sway [7-10]. Consequently, a patient who undertakes therapy for balance improvement should not use fixed support, because the control of postural sway is important for balance improvement.

We questioned whether fixed support was necessary to overcome the fear of falling. Suppose the perception of support is adequate as opposed to actual stable support. Perception of support could then be coupled with exercises that allow for the control of postural sway. We developed an apparatus that relies on the perception of support but allows the patient to encounter and control his or her sway characteristics. To provide proof-of-concept, we performed two studies with the novel balance rehabilitation and training apparatus. The purpose of study 1 was to determine whether this new apparatus significantly altered the biological postural control system. The purpose of study 2 was to document the benefits of balance training using the balance apparatus with an intervention case study.

\section{METHODS}

Figure 1 shows the balance rehabilitation and training apparatus used in these studies, which consists of a stable platform and a vertical structure that supports a movable carriage and two free-floating handles. In addition, horizontal handrails are mounted on each side of the platform. The free-floating handle consists of a $2.54 \mathrm{~cm}$-diameter

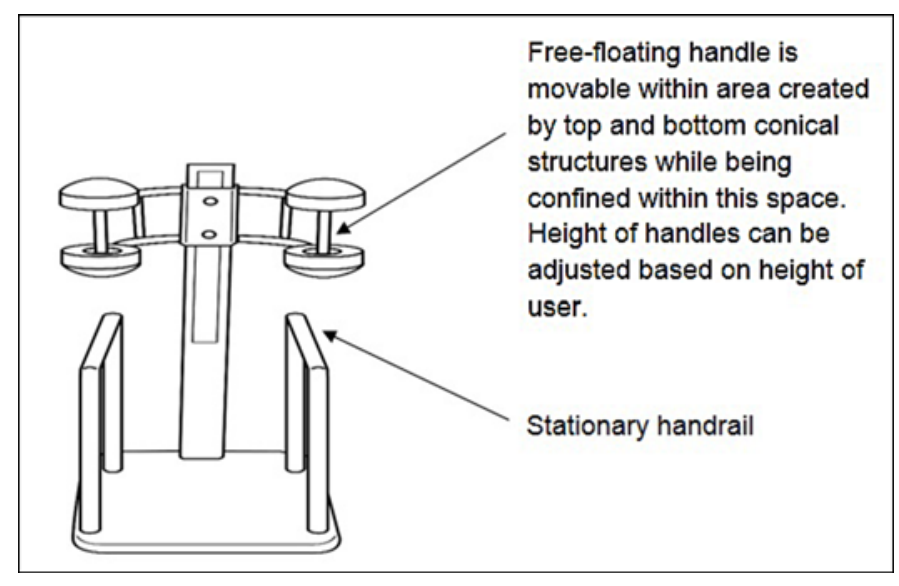

Figure 1.

Illustration of balance rehabilitation and training apparatus. Apparatus consists of stable platform with two stationary handrails and two free-floating handles to assist when training.

metal tube that the subject holds with his or her hand. The handle fits within the $9.0 \mathrm{~cm}$-diameter conical structures supported above and below the handle so that the handle is free-floating but constrained within the area of the conical structures. The handles provide three-dimensional movement, which includes $7.46 \mathrm{~cm}$ of displacement in the medial-lateral and anterior-posterior axes and $5.0 \mathrm{~cm}$ of displacement in the vertical axis. The diameter of the conical structures that create the displacement area was selected to allow for appropriate movement (i.e., postural sway) during balance exercises before the handles provide support for the user. Future design for the balance apparatus will include the ability to adjust the size of the displacement area to accommodate for individuals with different levels of functional balance or for various balance disorders. The subject was instructed to hold the freefloating handles so the handle was not touching any part of the apparatus. This allows the subject to experience unhindered postural sway while holding onto the handles. In addition, the otherwise free-floating handles become restrained when moved to the outside of the displacement area, thus limiting sway and providing support for the user. The height of the handles was adjusted based on the height of the user.

\section{Study 1}

Study 1 was a randomized, repeated-measures design in which six healthy adult subjects (mean age: $35.5 \mathrm{yr}$, 
range: 22 to $56 \mathrm{yr}$ ) with no history of neurological or musculoskeletal deficits were examined. Postural stability was assessed by the center of pressure (COP) data collected with a portable force platform (model 9286AA, Kistler Instrument Corp; Amherst, New York). Assessments were measured on the balance training apparatus during six different conditions: free-floating handles with eyes open and eyes closed (Figure 2(a)), freestanding with eyes open and eyes closed (Figure 2(b)), and stationary handrail with eyes open and eyes closed (Figure 2(c)). During the free-floating handles condition, the subject grasped the free-floating handles with the left and right hands, while during the stationary handrail condition, the subject lightly grasped the stationary handrails. Conversely, the subject did not hold onto anything during the freestanding condition (Figure 2). Subjects were tested barefooted, and all conditions were randomly administered to eliminate any order effect for testing. There was a minimum $5 \mathrm{~min}$ rest interval between each testing condition. During each condition, the subjects were instructed to stand as still as possible for $90 \mathrm{~s}$, during which the COP was recorded with a sample rate of $2 \mathrm{kHz}$.

\section{Study 2}

Study 2 was an intervention case study to determine whether performing balance training on the apparatus for 4 wk improved balance parameters in a generally healthy 63 yr-old individual with no significant medical issues or history of neurological or musculoskeletal deficits. Postural stability was assessed at baseline and then after training (posttraining) using the procedures described for study 1 . The subject reported to the laboratory $3 \mathrm{~d} / \mathrm{wk}$ for 4 wk to perform balance exercise training. Balance exercises were performed on the balance rehabilitation and training apparatus. A customized balance exercise program was prescribed by a licensed physical therapist. The

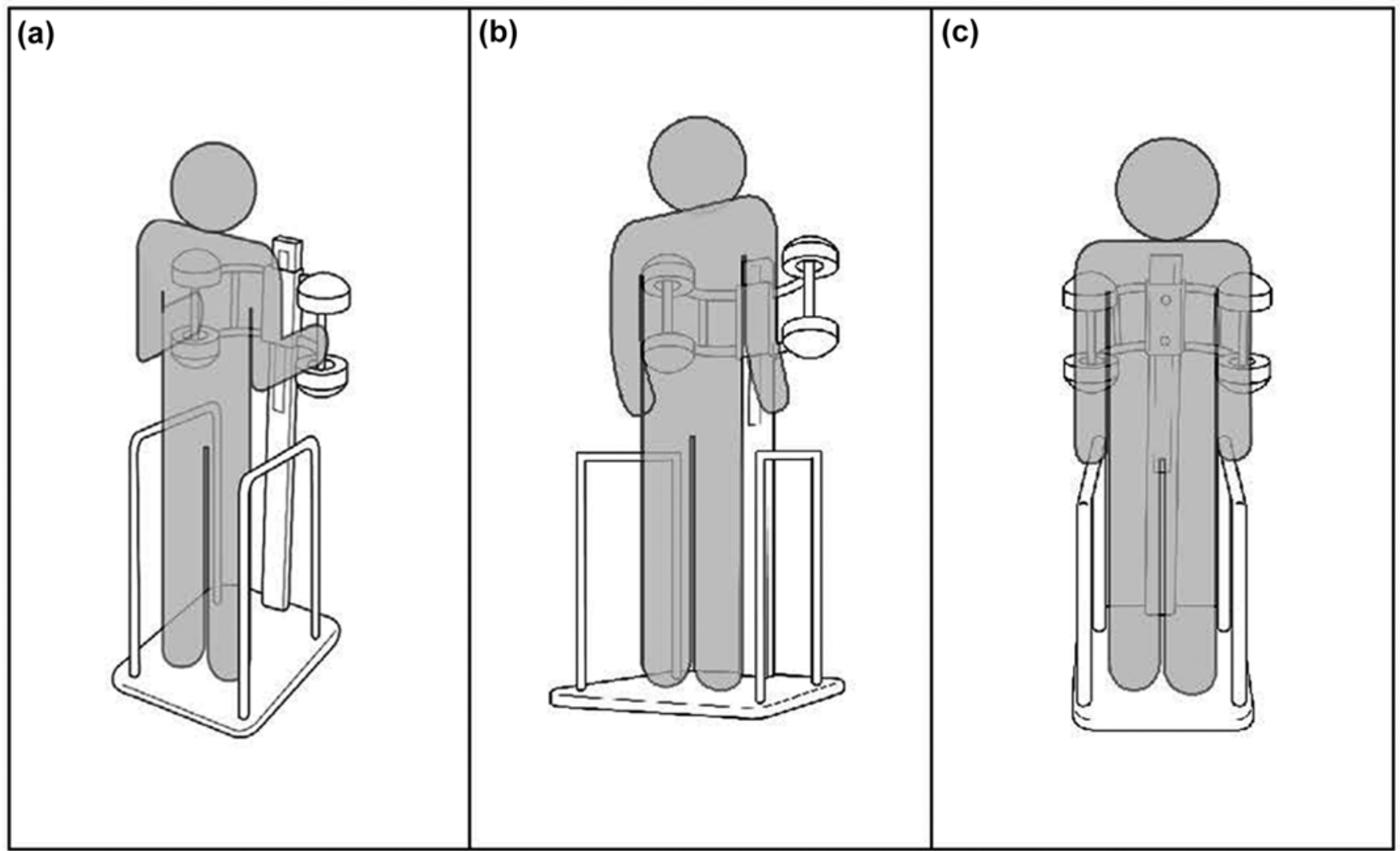

Figure 2.

Illustrations of different study conditions. (a) Subject stood while grasping free-floating handles. (b) Subject stood without any hand grasping (freestanding). (c) Subject stood while lightly grasping stationary handrail. 
difficulty of the balance exercises was established based on the functional balance ability of the subject. The same exercises in the same order were performed each week, and the exercises were changed to increase the difficulty at the beginning of each week. Individual exercises were performed while the subject held onto the free-floating handles and were approximately 30 to $60 \mathrm{~s}$ in duration, with each exercise session lasting approximately $15 \mathrm{~min}$. An exercise session consisted of approximately 20 exercises and included static standing, weight shifts (toe-heel, right-left), head turns (up-down, right-left), and stationary marching (with and without a foam pad placed on the platform). Exercises were performed with eyes open and eyes closed. This type of balance program is similar to what a physical therapist would prescribe for a patient to be completed independently at home or at a fitness center.

All analyses were performed in the MATLAB environment using custom-written computer codes (The MathWorks Inc; Natick, Massachusetts). The force platform data were down-sampled to $100 \mathrm{~Hz}$ and filtered using an eighth-order Butterworth low-pass filter with a cutoff frequency of $20 \mathrm{~Hz}$. The trajectory of the COP was calculated from the force platform data according to the recommendations of the manufacturer. To assess the amount of postural sway, path length (in millimeters) was measured, which was the total length that the subject's COP moved during $60 \mathrm{~s}$ of the $90 \mathrm{~s}$ trial. Sway area (in millimeters squared) was the area defined by the outermost movements of the COP in the medial-lateral and the anterior-posterior directions for a $60 \mathrm{~s}$ segment. A comprehensive explanation of these variables is detailed in the original work of Hufschmidt et al. [11].

To further quantify the changes in postural sway between baseline and posttraining, the detrended fluctuation analysis (DFA) method was used for the COP trajectory in both the anterior-posterior and the medial-lateral components of sway. This method has been extensively used for the investigation of many biological phenomena, including postural sway [12-14], and has been recently described by Tahayor et al. [15].

For study 1 , a repeated-measures one-way analysis of variance (ANOVA) was performed for all variables with three conditions: free-floating handles, freestanding, and stationary handrail. Data were analyzed separately for the eyes open and eyes closed conditions. Bonferroni post hoc analyses were completed when significant ANOVA results were found to examine where differences existed between conditions. A significance level was set at $p \leq$
0.05 . For study 2, percent change was calculated to assess the change in postural sway measurements from baseline to posttraining.

\section{RESULTS}

For study 1, the ANOVA results from the postural sway area, postural sway path length, and anterior-posterior complexity were significantly different between conditions. Further analyses with the Bonferroni comparisons revealed the free-floating handles and freestanding conditions were not significantly different from each other, which suggested that the free-floating handles on the balance training apparatus did not affect the normally occurring postural sway characteristics. As anticipated, postural sway during the stationary handrail condition was significantly lower ( $p \leq$ 0.05 ) than the free-floating handles and freestanding conditions. Postural sway area and path length for the eyes open condition were 61 and 38 percent lower $(p \leq 0.05)$, respectively, during the stationary handrail condition than the free-floating handles condition (Figures 3-4). Similarly, for the eyes closed condition, postural sway area and path length were 40 and 48 percent lower $(p \leq 0.05)$, respectively, during the stationary handrail condition (Figures 34). In both cases, the free-floating handles condition was not significantly different than the freestanding condition (Figures 3-4). Additionally, the dynamic complexity of the

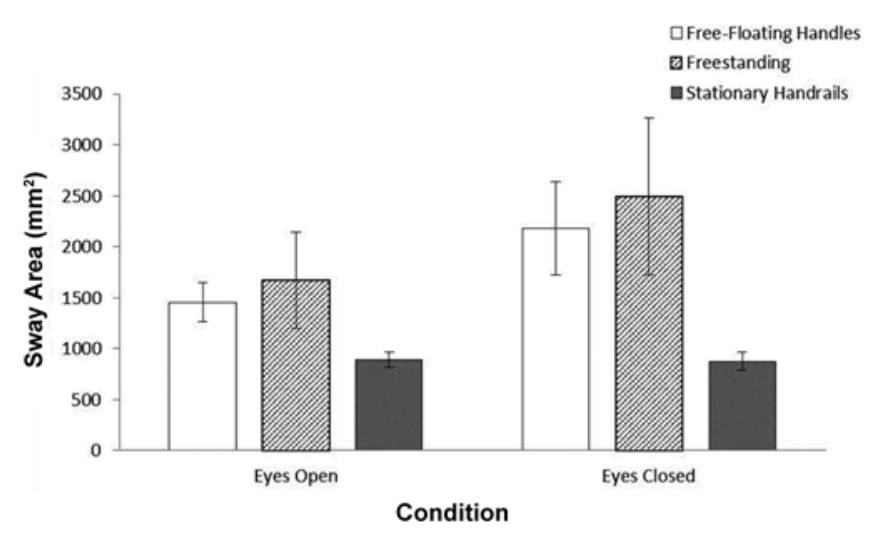

Figure 3.

Postural sway area. Note similarity in postural sway area for both free-floating handles and freestanding conditions with eyes open and eyes closed. Significant difference exists between stationary handrail condition and both free-floating handles and freestanding conditions. 


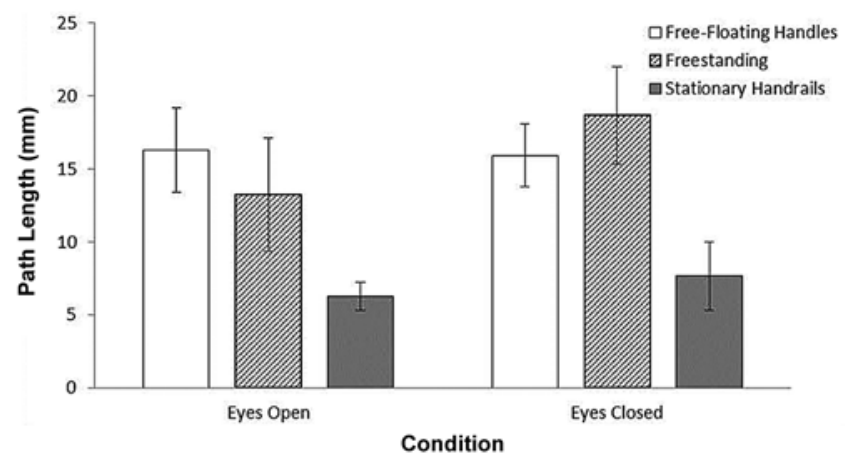

Figure 4.

Postural sway path length. Note similarity in postural sway path length for both free-floating handles and freestanding conditions with eyes open and eyes closed. Significant difference exists between stationary handrail condition and both free-floating handles and freestanding conditions.

postural sway signal was not affected during the free-floating handles and freestanding conditions but was affected by the stationary handrail condition $(p=0.001)$. Figure 5 shows these results.

For study 2, it was shown that improvements in postural stability can be obtained with 4 wk of training. The measurements of postural sway were improved in response to training during the eyes closed condition, the most challenging postural condition examined (Table). Sway area, lateral sway, and anterior sway decreased by $28.63,2.30$, and 27.60 percent, respectively, in response to training (Table). The improvement in postural stability parameters is supported by the change in the complexity of postural control. The rambling component was decreased by 17 percent (from 0.58 to $0.48 \mathrm{~mm}$ ), and the trembling component was increased by 214 percent (from 0.11 to $0.25 \mathrm{~mm}$ ).

\section{DISCUSSION}

The purpose of developing the balance rehabilitation and training apparatus was to provide a useful tool to allow individuals with fear of falling to effectively and safely perform balance exercises and to provide individuals an environment for training in which the normally occurring dynamics of the postural control system are not disrupted. Common practice for therapists is to encourage individuals to perform independent balance exercises

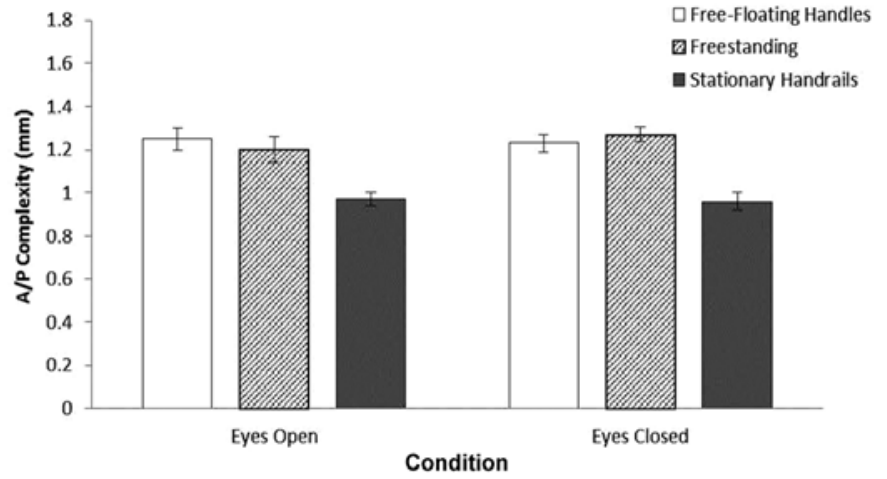

Figure 5.

Anterior-posterior (A/P) complexity. Note similarity in A/P complexity for both free-floating handles and freestanding conditions with eyes open and eyes closed. Significant difference exists between stationary handrail condition and both free-floating handles and freestanding conditions.

while not holding onto a stable object. This allows them to experience postural sway, which is an important aspect for improving balance. The seminal works of Holden et al. [7] and Jeka and Lackner [8-9] introduced the importance of haptic information in the context of postural control. Haptic information refers to the kinesthetic and cutaneous input from mechanoreceptors within the muscles, joints, and skin of the fingers and arms. This information provides an orientation sense by providing important feedback, which helps minimize postural sway. Numerous studies have shown that haptic supplementation provided by fixed or mobile "touch support" enhances postural stability in young and elderly subjects [7-10]. Whereas this notion may be instrumental for stabilizing the body, it may not present an ideal situation for training the systems that control balance. Training is most effective when presented in the most naturally occurring environment, and limiting the normally occurring sway may in fact hinder rather than facilitate the learning process. Ideally, the free-floating handles would not provide haptic information such that they enhance postural stability. This was supported in study 1, because postural sway area and path length during the freestanding and freefloating handles conditions were not significantly different. In contrast, holding onto the stationary handrail produced between 40 and 61 percent less postural sway area and postural path length than the free-floating handles and freestanding conditions. These data support the notion 
Table.

Comparison of postural sway measurements from baseline to posttraining during eyes closed condition. Each postural sway variable represents one aspect of sway characteristics measured before and after training. Note improvements in these parameters following training with balance rehabilitation and training apparatus.

\begin{tabular}{lccc}
\hline \multicolumn{1}{c}{ Postural Sway Measurement } & Baseline & Posttraining & Improvement (\%) \\
\hline Sway Area $\left(\mathrm{mm}^{2}\right)$ & 5.57 & 3.97 & 28.63 \\
Lateral Sway (SD) & 0.79 & 0.77 & 2.30 \\
Anterior Sway (SD) & 0.58 & 0.42 & 27.60 \\
Rambling Component of Sway (mm) & 0.58 & 0.48 & 17.10 \\
Trembling Component of Sway (mm) & 0.11 & 0.25 & 214.00 \\
\hline SD = sandard deviation.
\end{tabular}

$\mathrm{SD}=$ standard deviation.

that performing balance exercises when you have not disrupted the normal "control" systems would be much more effective than when performing training while holding onto a fixed or mobile support that may impose an artificial constraint on the postural control system. While the sway outcomes were not different between the freestanding and free-floating handles conditions, it is unclear whether other haptic information was provided under the conditions of this study.

Another important factor of balance training in older individuals is fear of falling. It is well documented that individuals often choose not to perform activities or exercises necessary to improve functional balance simply due to their fear of falling [6]. More research is needed to determine whether using the free-floating handles during balance training can minimize fear of falling and improve functional balance.

Study 2 showed improvements in postural stability as a result of $4 \mathrm{wk}$ of balance training using the balance rehabilitation and training apparatus. The postural sway area was decreased by 28 percent, illustrating enhanced postural stability after training. This suggests the apparatus could be an effective tool for improving functional balance and does not attenuate any potential training effect when coupled with other training devices. In addition, this could also provide a safe and effective way to perform balance exercises independent of a therapist at home or in a fitness center. The dynamic complexity of postural sway was evaluated before and after training. During normal standing, the amount of sway is coordinated by both the descending control from the cortex as well as the segmental or lower-level control (spinal cord control) from the proprioceptors of the foot and leg musculature. The rambling component of the DFA refers to the cortical control of the sway parameters, whereas the trembling component refers to the proprioceptive control of the sway parameters [15]. In the eyes closed condition, the rambling component decreased by 17 percent and the trembling component increased by 214 percent after training. This suggests a positive shift in the control of the COP from the higher central nervous system centers toward the lower levels (reflex and proprioceptive systems). The role of training is to shift this control to the faster and more dynamic proprioceptive mechanisms, which because of their locus in the spinal cord, respond much quicker to sudden disturbances in the postural system, e.g., during fall-avoidance episodes. Our data showed that using the balance rehabilitation and training apparatus during the $4 \mathrm{wk}$ training program led to improved balance characteristics for this subject.

We acknowledge that there are limitations to the studies, including the small number of subjects and the fact that the subjects were not balance impaired. A long-term training study would also be beneficial and should include individuals with fear of falling. Additional information should be collected regarding the subjective assessment of fear of falling and the potential subjective benefits from using the apparatus. The findings from these proof-ofconcept studies warrant additional investigations to assess the feasibility of the balance rehabilitation and training apparatus in individuals that have traumatic brain injury, stroke, amputation, visual impairment, vertigo, inner-ear damage, concussion, war-related injuries, neuromuscular disease, and movement disorders.

\section{CONCLUSIONS}

The results of these investigations support the functionality of the balance rehabilitation and training apparatus as an effective tool to help individuals safely and independently perform balance exercises. The apparatus 
could help a large number of balance-impaired individuals improve their functional balance and potentially decrease falling and fall-related injuries. Also, the apparatus could be an important component to help implement the fall-prevention strategies that have been identified and to minimize the huge economic burden on societies and healthcare systems throughout the world.

\section{ACKNOWLEDGMENTS}

\section{Author Contributions:}

Study concept and design: D. M. Koceja, J. S. Greiwe.

Analysis and interpretation of data: D. M. Koceja.

Drafting of manuscript: D. M. Koceja, J. S. Greiwe.

Financial Disclosures: Dr. Greiwe is the owner of Balancemetrics, which is developing the balance rehabilitation and training apparatus (Freesway Balance Trainer). Provisional and nonprovisional patent applications have been submitted that describe the apparatus and method of using the apparatus. This article is descriptive in nature and does not make any claims regarding the safety or effectiveness of the balance rehabilitation and training apparatus. The results presented in this article pertain to the feasibility of the apparatus in the population assessed. Dr. Koceja does not hold any equity or ownership in Balancemetrics.

Funding/Support: This material was unfunded at the time of manuscript preparation. No financial support was provided by Balancemetrics. The balance rehabilitation and training apparatus was provided to the investigators for research purposes.

Additional Contributions: We thank Whitney Ogle, Behdad Tahayori, and Koichi Kitano for their execution of the study and Tony Thompson for his technical support.

Institutional Review: The rationale and procedures for these studies were approved by the Indiana University Institutional Review Board. Participant Follow-Up: The authors do not plan to notify the subjects of the publication of this study because of a lack of contact information.

\section{REFERENCES}

1. Administration for Community Living. Administration on Aging (AoA): Projected future growth of the older population [Internet]. Washington (DC): Administration for Community Living; 2008 Aug 14. Available from: http:// www.aoa.gov/Aging_Statistics/future_growth/

future growth.aspx

2. Kochanek KD, Xu J, Murphy SL, Miniño AM, Kung HC. Deaths: Final data for 2009. Natl Vital Stat Rep. 2011; 60(3):1-116. [PMID:24974587]

3. Englander F, Hodson TJ, Terregrossa RA. Economic dimensions of slip and fall injuries. J Forensic Sci. 1996; 41(5):733-46. [PMID:8789837]
4. Stevens JA, Corso PS, Finkelstein EA, Miller TR. The costs of fatal and non-fatal falls among older adults. Inj Prev. 2006;12(5):290-95. [PMID:17018668] http://dx.doi.org/10.1136/ip.2005.011015

5. Rose DJ. Reducing the risk of falls among older adults: The Fallproof Balance and Mobility Program. Curr Sports Med Rep. 2011;10(3):151-56. [PMID:21623303] http://dx.doi.org/10.1249/JSR.0b013e31821b1984

6. Tinetti ME, Powell L. Fear of falling and low self-efficacy: A case of dependence in elderly persons. J Gerontol. 1993; 48(Spec No):35-38. [PMID:8409238] http://dx.doi.org/10.1093/geronj/48.Special_Issue.35

7. Holden M, Ventura J, Lackner JR. Stabilization of posture by precision contact of the index finger. $\mathrm{J}$ Vestib Res. 1994;4(4):285-301. [PMID:7921347]

8. Jeka JJ, Lackner JR. Fingertip contact influences human postural control. Exp Brain Res. 1994;100(3):495-502. [PMID:7813685] http://dx.doi.org/10.1007/BF02738408

9. Jeka JJ, Lackner JR. The role of haptic cues from rough and slippery surfaces in human postural control. Exp Brain Res. 1995;103(2):267-76. [PMID:7789434] http://dx.doi.org/10.1007/BF00231713

10. Albertesen, IM, Temprado, JJ. Supplémentation haptique et contrôle postural chez les personnes âgées: Revue et perspectives pour les technologies d'assistance [Haptic supplementation and postural control in the elderly: review and perspectives for assistive technologies]. Sci Motricite. 2011;74:101-17. French. http://dx.doi.org/10.1051/sm/2011118

11. Hufschmidt A, Dichgans J, Mauritz KH, Hufschmidt M. Some methods and parameters of body sway quantification and their neurological applications. Arch Psychiatr Nervenkr. 1980;228(2):135-50. [PMID:7458637] http://dx.doi.org/10.1007/BF00365601

12. Duarte M, Zatsiorsky VM. On the fractal properties of natural human standing. Neurosci Lett. 2000;283(3):173-76. [PMID:10754215] http://dx.doi.org/10.1016/S0304-3940(00)00960-5

13. Zatsiorsky VM, Duarte M. Instant equilibrium point and its migration in standing tasks: Rambling and trembling components of the stabilogram. Motor Control. 1999;3(1):28-38. [PMID:9924099]

14. Zatsiorsky VM, Duarte M. Rambling and trembling in quiet standing. Motor Control. 2000;4(2):185-200. [PMID:11500575]

15. Tahayor B, Riley ZA, Mahmoudian A, Koceja DM, Hong SL. Rambling and trembling in response to body loading. Motor Control. 2012;16(2):144-57. [PMID:22357153]

Submitted for publication March 24, 2014. Accepted in revised form June 16, 2014. 
JRRD, Volume 51, Number 8, 2014

This article and any supplementary material should be cited as follows:

Koceja DM, Greiwe JS. Novel balance rehabilitation and training apparatus to improve functional balance. J Rehabil Res Dev. 2014;51(8):1331-38.
http://dx.doi.org/10.1682/JRRD.2014.03.0082

Au SUBMissions SCREENE or. $\checkmark$ iThenticate

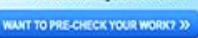

HEMBER sing

CROSSREF.ORG

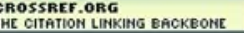

